\title{
CAN WE CHECK BY HIGH-DISPERSION ANALYSES STARS BELONGING TO EGGEN'S MOVING GROUPS?
}

\author{
L. E. PASINETTI
}

Milano-Merate Observatory, Italy

\begin{abstract}
We describe the results of analysis of the giant star HD 6497, which belongs to the Arcturus group.
\end{abstract}

The present discussion concerns a giant star (HD 6497) (Cayrel de Strobel and Pasinetti, 1975), which belongs to the Arcturus group according to Eggen (1974).

This star had been found to be super-metal-rich from narrow-band photometry (Williams, 1971) and metal deficient from the spectroscopic detailed analysis (Cayrel de Strobel, 1966).

We have performed a new detailed analysis employing a very good method proposed by Foy (1971) for reducing the spectrograms. This method allows us to obtain a curve of growth enriched in weak lines. With such a curve (Figure. 1), we have obtained without any doubt a normal iron abundance in respect to the Sun, in agreement with the results obtained from the Geneva photometry (Grenon, 1972), and from Hansen and Kjaergaard

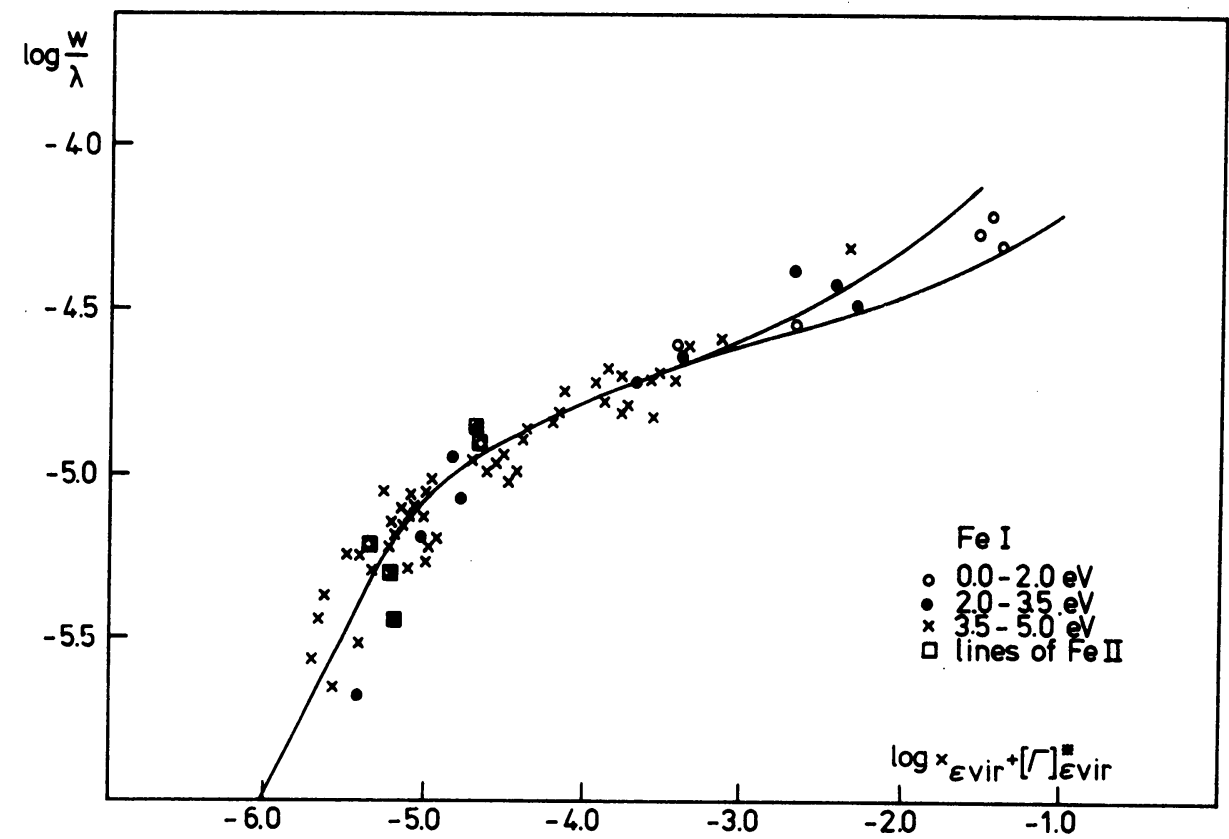

Fig. 1. Curve of growth of Fe I enriched in weak lines. 
(1971). Consequently this new determination has slightly changed the physical parameters which we determine from the neutral and ionized iron lines.

Two remarks are necessary on this result:

- The first remark concerns the spectroscopic techniques: it is easy to understand this disagreement with the previous spectroscopic analysis, taking into account that in the former analysis Cayrel de Strobel has not put enough weak lines on the linear part of the curve of growth. Therefore the possibility exists to draw the curve of growth in two different ways (Figure 2): small iron deficiency and high microturbulence or normal metal abundance and low microturbulence.

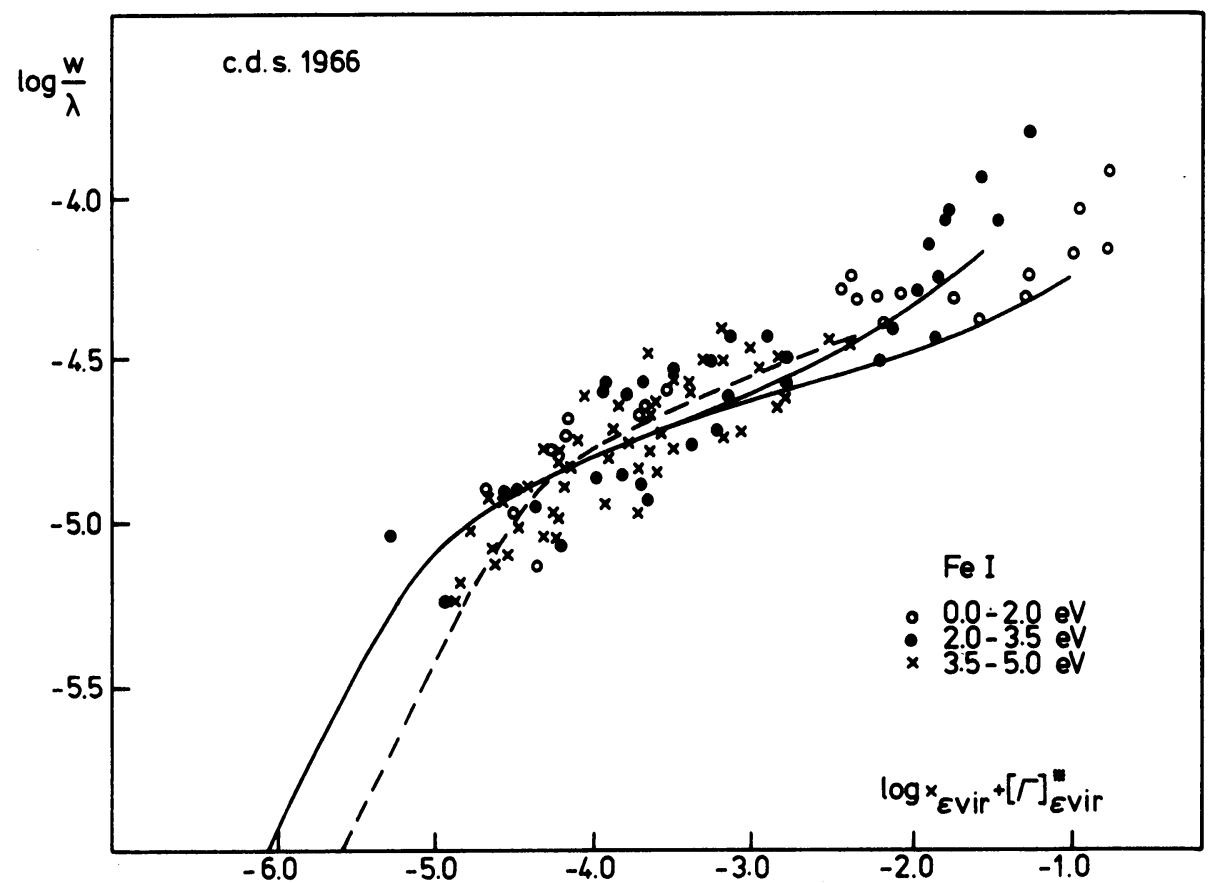

Fig. 2. Curve of growth of Fe I obtained from the material of Cayrel De Strobel (1966).

- The secorid remark regards an interesting problem of galactic structure. Eggen had considered the previous iron abundance i.e. the metal deficiency of this star, as an indicator of the metal abundance of the Arcturus group. We know (Mäckle et al., 1975) that Arcturus is surely a metal-deficient star. Therefore several possibilities arise:

Our star may not belong to the Arcturus group or Arcturus itself may not belong to the group. The group may not be homogeneous in chemical composition or, finally, some doubt may arise also on the existence of the Arcturus group itself, at least as it has been given by Eggen.

We have begun at Victoria Observatory an observational programme for testing other stars of the Arcturus group from a spectroscopic point of view. 


\section{References}

Cayrel de Strobel, G.: 1966, Ann. Astrophys. 29, 413.

Cayrel de Strobel, G. and Pasinetti, L. E.: 1975, Astron. Astrophys. 43, 127.

Eggen, O. J.: 1974, Publ. Astron. Soc. Pacific 86, 162.

Foy, R.: 1971, Astron. Astrophys. 11, 89.

Grenon, M.: 1972, private communication.

Hansen, L. and Kjaergaard, P.: 1971, Astron. Astrophys. 15, 123.

Mäckle, R., Holweger, H., Griffin, R. and Griffin, R.: 1975, Astron. Astrophys. 38, 239.

Williams, P. M.: 1971, Monthly Notices Roy. Astron. Soc. 153, 171.

\section{DISCUSSION}

Williams: Did you use the same spectroscopic plates as in your previous analysis? As to metal abundances of Group stars: I have used narrow-band photometry to investigate abundances of giants assigned to various kinematic groups and apart from finding Hyades (cluster)-like abundances for several stars assigned to the Hyades group, have not found the older groups to be chemically homogeneous.

Pasinetti: No, we have used new spectroscopic plates, but with same dispersion and resolution as in the previous analysis. Regarding the comment: Eggen has given only one abundance value as representative of all the group, precisely the iron abundance of HD 6497. 